\title{
RECREATING CULTURAL HERITAGE ENVIRONMENTS FOR VR USING PHOTOGRAMMETRY
}

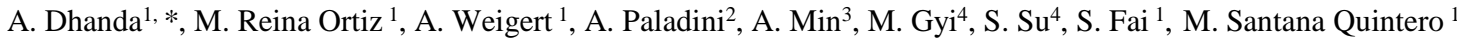 \\ ${ }^{1}$ Carleton Immersive Media Studio (CIMS), Carleton University, Ottawa, Canada - (adhanda, aweigert, sfai)@ cims.carleton.ca, \\ (miquel.reinaortiz, mario.santana)@ carleton.ca \\ ${ }^{2}$ Raymond Lemaire International Centre for Conservation (RLICC), KU Leuven, Leuven, Belgium - \\ alice.paladini@student.kuleuven.be \\ ${ }^{3}$ Department of Archaeology and National Museum, Bagan, Myanmar \\ ${ }^{4}$ Mandalay Technological University, Mandalay, Myanmar
}

\section{Commission II}

KEY WORDS: Virtual Reality, Photogrammetry, Physically Based Rendering, Cultural Heritage, Bagan

\begin{abstract}
:
In this paper, we propose a workflow for recreating places of cultural heritage in Virtual Reality (VR) using structure from motion (SfM) photogrammetry. The unique texture of heritage places makes them ideal for full photogrammetric capture. An optimized model is created from the photogrammetric data so that it is small enough to render in a real-time environment. The optimized model, combined with mesh maps (texture maps, normal maps, etc.) looks like the original high detail model. The capture of a whole space makes it possible to create a VR experience with six degrees of freedom (6DoF) that allows the user to explore the historic place. Creating these experiences can bring people to cultural heritage that is either endangered or too remote for some people to access. The workflow described in this paper will be demonstrated with the case study of Myin-pya-gu, an 11th century temple in Bagan, Myanmar.
\end{abstract}

\section{BACKGROUND AND RELATED WORKS}

\subsection{Virtual Reality}

VR can be presented in a $3 \mathrm{DoF}$ or $6 \mathrm{DoF}$ system. In a $3 \mathrm{DoF}$ VR system the user's head movements (rotation) are tracked. This is what is known as 'passive VR'; where the user can look but cannot control their physical movements in the space. In a $6 \mathrm{DoF}$ VR system the user's head and body movements are tracked (rotation and translation) (Google Developers, 2018). 6DoF VR is also known as 'active VR'. Slater and Wilbur, 1997 stated that correlation between the user movements and the virtual movements is a crucial factor that affects how a user feels presence in a virtual space. Therefore, a $6 \mathrm{DoF}$ experience has a greater potential in transporting the user to a location. Additionally, 6DoF systems are becoming more affordable, and will soon be useable without an external computer (Oculus, 2018).

Many current VR experiences for cultural heritage are either passive or active experiences that use a traditional video game workflow (Kersten et al., 2017; Graham et al., 2018; Debailleux et al, 2018). This traditional workflow uses assets that are manually 3D modelled and then textured using orthophotos or approximations of the real materials (Kersten et al., 2017; Debailleux et al, 2018). Slater and Wilbur, 1997 state that Vivid is one of the four factors that affects immersion in VR. Vivid includes things such as the visual and colour resolution, and the quality of the displays. We believe that a fully photogrammetric approach can lead to more realistic results when compared to a traditional approach; a more vivid illusion.

\subsection{Photogrammetry in Game Environments}

Computational advances in the last decade have made it possible to render more complex geometry in real-time game environments. The Vanishing of Ethan Carter and The Talos Principle - two games that use photogrammetry to build the set pieces - were released in late 2014 (Croteam, 2014; Statham, 2018). The team from DICE used photogrammetry and physically based rendering (PBR) to create the world for 2015's Star Wars Battlefront (Statham, 2018). PBR is a way of rendering the accurately represents how light interacts with materials and surfaces (McDermott, 2018). Since 2016, several VR experiences have used photogrammetry to recreate spaces: Valve's Destinations, Realities by Realities.io, and Masterworks $V R$ by CyArk (Valve, 2016; Realities.io, 2016; CyArk, 2018). However, there is only one experience - that the authors are aware of - that uses photogrammetry and PBR to fully recreate a space in VR: Nefertari: Journey to Eternity by Reality Virtual, a recreation of the tomb of Queen Nefertari in Egypt (Curiosity Stream et al, 2018).

Hellman and Lahti, 2018 discussed a basic photogrammetry to VR workflow, but focused more on processing the photogrammetry than on optimizing it. Also, they did not use mesh maps (other than the texture map). Lachambre et al, 2017 introduced a photogrammetry to game engine workflow, but focused more on objects as opposed to whole sites. FernándezPalacios et al., 2017 used photogrammetry to recreate Etruscan tombs in VR. However, they focused on the development of the experience VR as opposed to the whole workflow, and the experiences used a system that was close to $6 \mathrm{DoF}$ but did not allow full interaction.

\footnotetext{
* Corresponding author
} 


\section{WORKFLOW AND CASE STUDY}

Bagan is in the centre of Myanmar in Southeast Asia. The archaeological site of Bagan was the capital of the first kingdom of Myanmar from 1044 to 1287 A.D. The site contains over 3000 temples and monuments, including the $11^{\text {th }}$ century temple Myinpya-gu. The solid core temple was constructed with brick masonry. The core of Myin-pya-gu has walls and vaults that are covered in mural paintings depicting tangent circle patterns, stupas, lotus flowers, jataka panels, and Buddha figures. According to Pierre Pichard, $30 \%$ of the mural paintings on the vault and $10 \%$ of the ones on the walls remained. The inner wall of the temple core is lined with Buddha statues that had been damaged over time (Pichard, 1995). Due to its condition, the temple is no longer open for public viewing, which makes it a candidate for VR reconstruction.

The inner core of the temple was captured using photogrammetry and laser scanning during the workshop 'Conserving Bagan's Built Heritage for Posterity' in May, 2018. The workshop was held in conjunction with Carleton University, Mandalay Technological University, and Myanmar's Department of Archaeology, National Museum and Library (DoA).

The photogrammetry to VR workflow outlined in Figure 1 is discussed in the following sections.

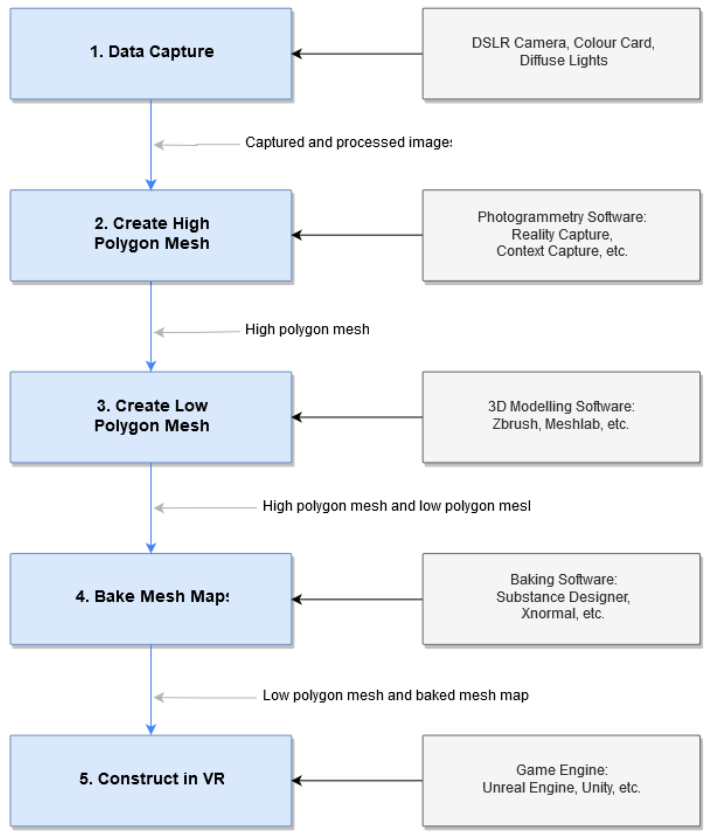

Figure 1. Photogrammetry to VR workflow

\subsection{Data Capture}

The SfM capture process for VR is similar to measurement for a metric survey. However, special attention needs to be paid to lighting and colour. It is critical that the images are lit with diffuse, even lighting while using a colour calibration chart to ensure accurate colour capture (Figure 2 top). Colour is discussed in more detail in Section 2.4.1.

It is also important to remember that all surfaces are important when capturing for VR, including ones that might not be considered during a metric survey. For example, the photogrammetric documentation of Myin-pya-gu was originally intended for metric purposes. Due to this, no photographs were taken of the temple floor (Figure 2 bottom). The missing geometry was filled in with information from the laser scans. The results could be improved if VR had been considered during the documentation.

The photogrammetry of the interior of Myin-pya-gu was acquired using a Nikon D750 with a $28 \mathrm{~mm}$ Nikkor lens at a nominal GSD of around $0.5 \mathrm{~mm}$.

\subsection{Create High Polygon Mesh ('High poly')}

The high poly mesh is created by processing the photogrammetry in a typical SfM pipeline: feature extraction and matching, selfcalibrating bundle adjustment, dense image matching, dense point cloud generation, and mesh generation. Agisoft Photoscan, Bentley Context Capture, and Reality Capture were considered for this part of the workflow. Reality Capture was used because of its processing speed, and the output mesh size is not limited by the user's RAM (Remondino et al, 2017). If the site is too large the space can be divided and processed in section that are aligned using ground control points (GCPs). For a local coordinate system, the base point is usually set to an arbitrary large value (ex, 1000, 1000, 1000). This point should be set as the zero point $(0,0,0)$ - and the other points shifted accordingly - before using the points to register the photogrammetry together. This will make it easier to work with the data in the game engine.

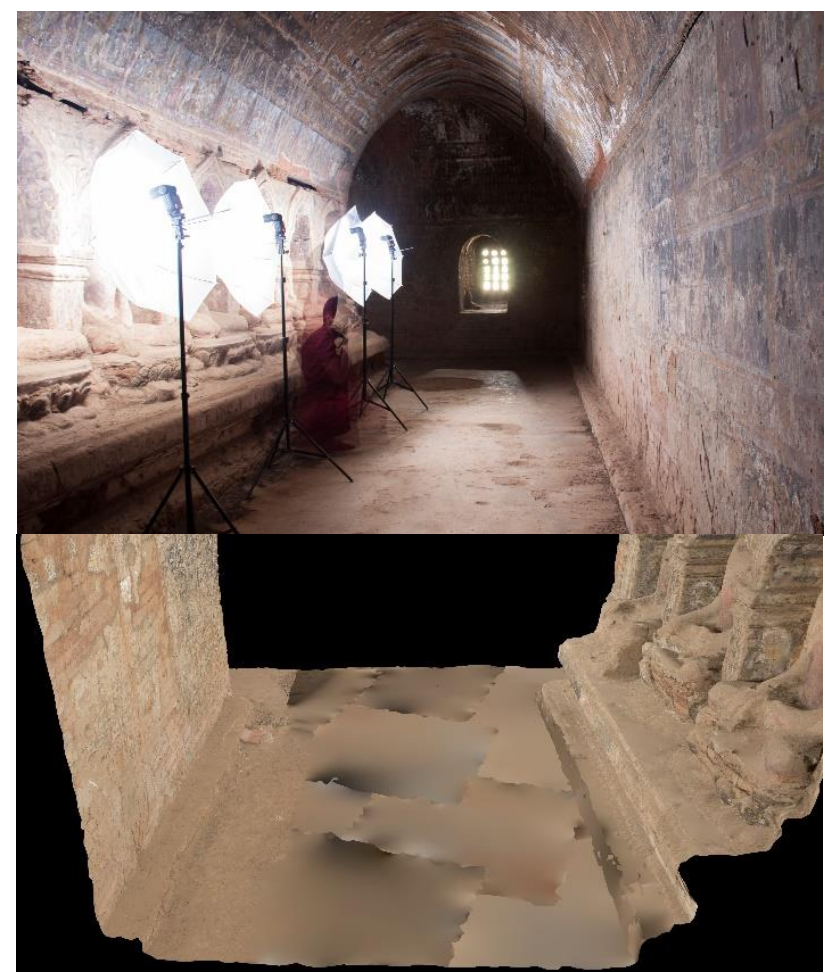

Figure 2. The photogrammetric lighting setup (top), and the missing data (bottom)

\subsection{Create Low Polygon Mesh ('Low poly')}

The high poly mesh is too large to render in a real-time environment, which is why a reduced version - the low poly mesh - is created. The mesh is decimated in two stages. First, the high poly mesh is divided into pieces and decimated into a 'medium poly' mesh in the photogrammetric software. This smaller mesh - usually between 10 and 30 million polygons - is used to bake the mesh maps (see Section 2.4) and is easier to work with when optimally decimating the mesh into a low poly mesh. The low poly mesh is made in a 3D modelling program like Meshlab or Zbrush (Kuzmin, 2018). 


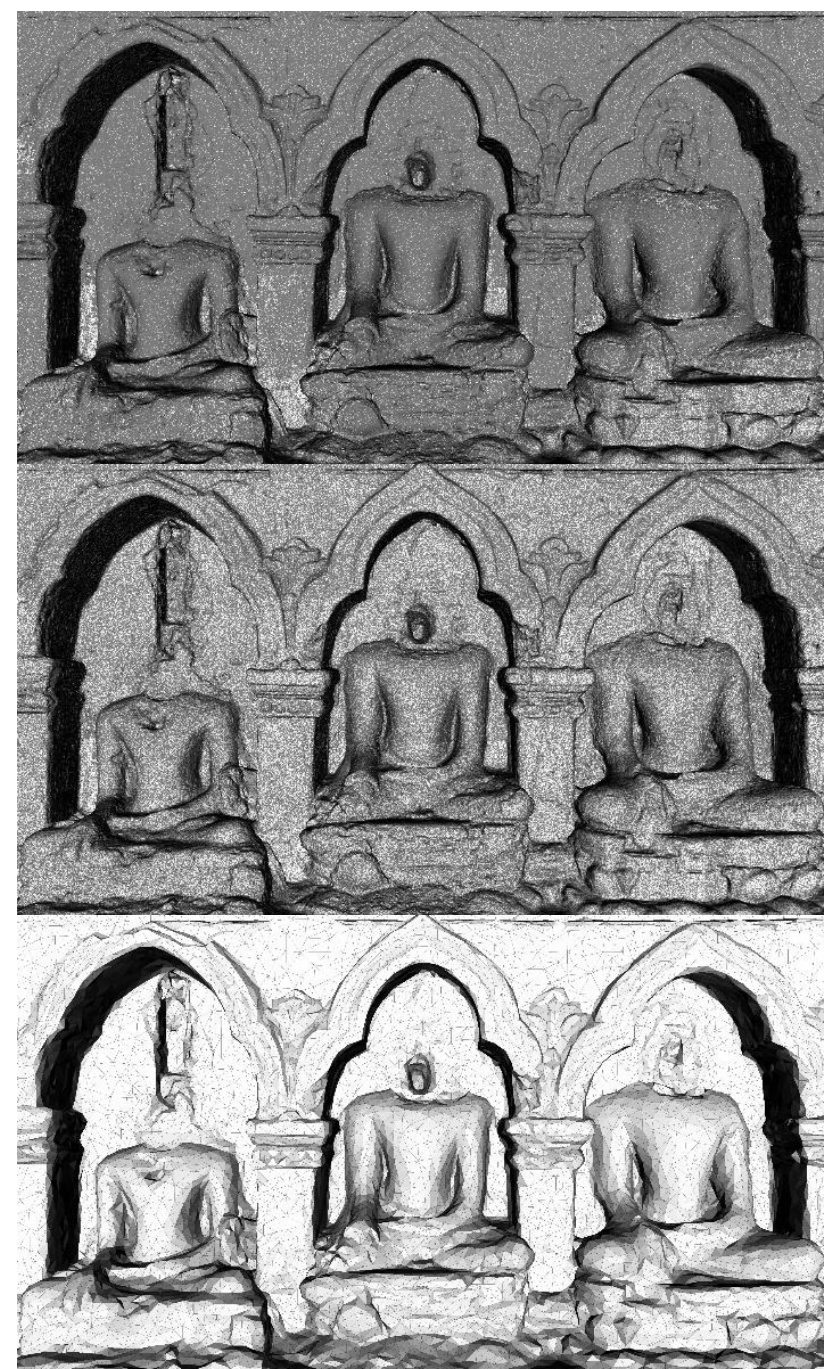

Figure 3. The different stages of decimation applied to a piece of the Myin-pya-gu model: 44 million polygons (top), 12 million polygons (middle), 60 thousand polygons (bottom)
The Quadratic Edge Collapse Decimation algorithm in Meshlab was used to make the low poly models for the Myin-pya-gu model (Cignoni, 2008). Figure 3 shows the decimation of a piece of the model from 44 million polygons (high poly) to 12 million polygons (medium poly), and then to 60 thousand polygons (low poly). Despite the high level of decimation, the low poly model does not deviate from the medium poly model by more than $\pm 1.5 \mathrm{~cm}$ (Figure 4 ).

\subsection{Bake Mesh Maps}

The mesh maps enable the low poly mesh to realistically resemble the high poly mesh. There are many kinds of mesh maps, but there five main maps used in PBR: The texture (or albedo colour map), the normal map, the ambient occlusion map, the roughness map, and the metallic map. The texture map holds all the colour information for the mesh. Normal maps use RGB values to encode surface normal directions. When a normal map is baked from a high poly to a low poly model it creates an impression of depth on the low poly model when it is lit; faking the detail of the high poly model. Ambient occlusion approximates the inner shadows of objects when they are under diffuse lighting. The roughness map identifies the irregularities in a surface that cause light to scatter diffusely. And lastly, the metallic map defines what part of the material are metal (McDermott, 2018). Figure 5 shows an example of a texture, normal, and ambient occlusion map from the Myin-pya-gu model that were baked using Allegorithmic's Substance Designer. The roughness and metallic values were approximated with constants based on known values of similar materials. In the future, these values will be captured on site so that each material can be represented accurately.

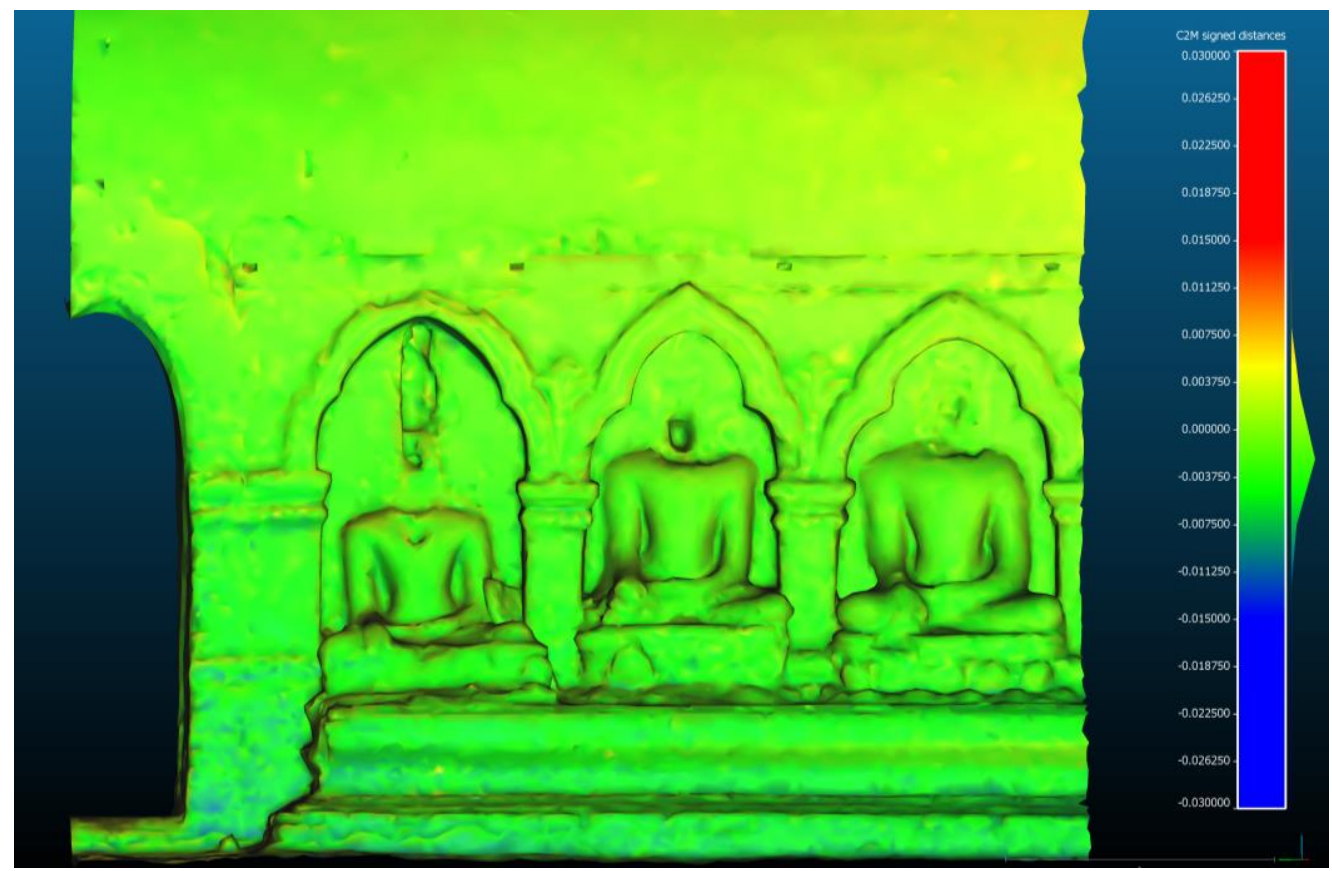

Figure 4 . The deviation between the low poly and the medium poly models (in metres) 


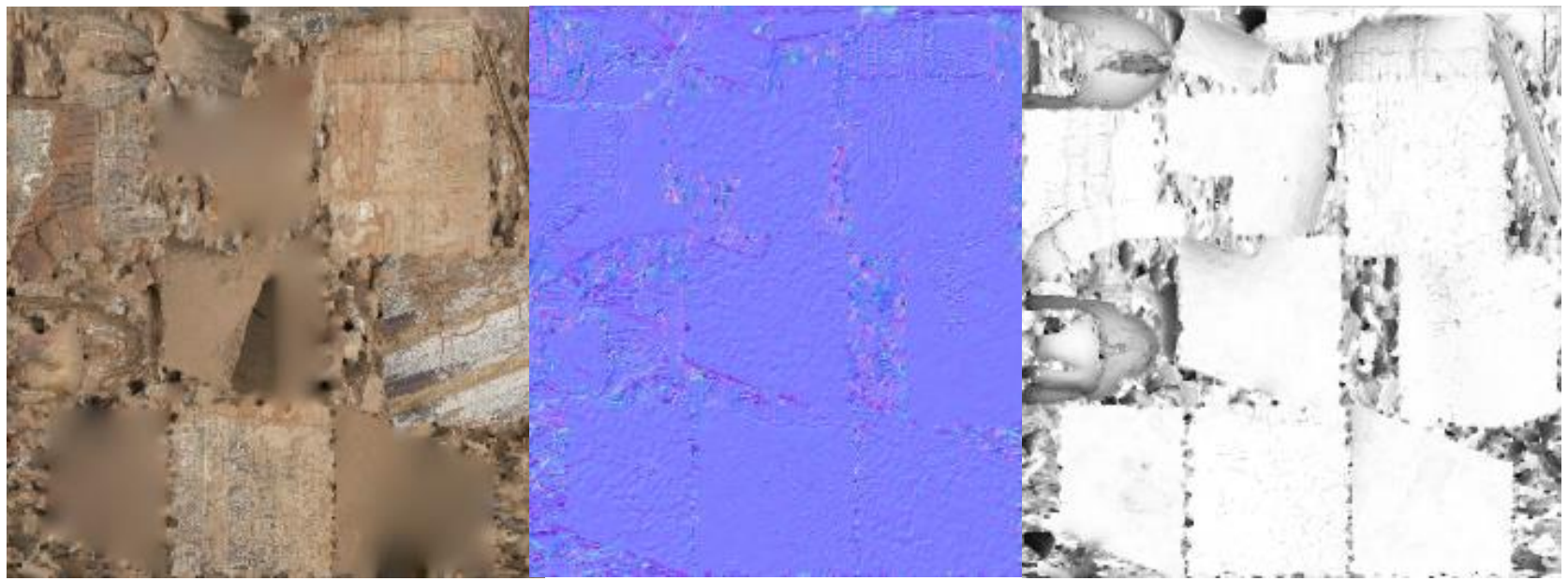

Figure 5. Mesh maps from the Myin-pya-gu model: a texture map (left), normal map (middle), and ambient occlusion map (right)

Colour Fidelity in VR: As mentioned in Section 1.1 colour resolution is one of the factors that plays a role in VR immersion (Slater and Wilbur, 1997). So, more accurate colour, would lead to increased user immersion within the virtual space. There are several colour spaces within the RGB colour model (Figure 6). The game engines available for the public (Unreal Engine, Unity, Cryengine, etc.) can only render texture maps with colours in the sRGB colour space. This limits the range of colours that can be rendered when compared to human perception. A colour space like ProPhoto RGB would lead to more accurate colour reproduction.

Shadows and de-lighting of texture maps also affects colour fidelity, however it will not be discussed in this paper (see Lachambre et al., 2017).

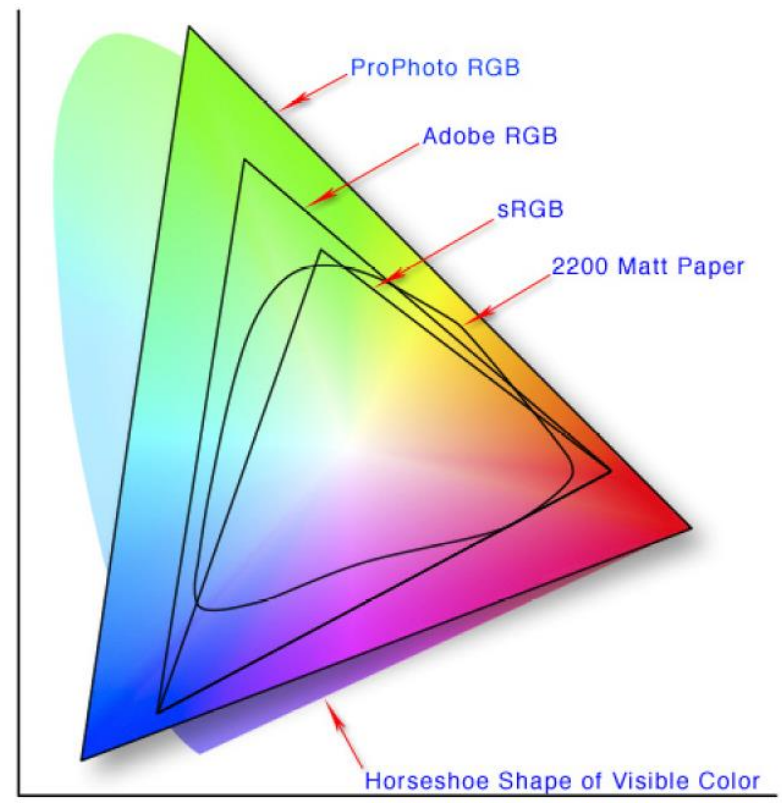

Figure 6. Colour spaces within the RGB colour model (Schewe, 2018)

\subsection{Construct in VR}

As the last step, the pieces of the model are placed in a video game engine. Because the pieces are all in the same coordinate system, they should align together. Once the pieces and their mesh maps are put together, the model is lit, and the VR controls are added.

The VR of Myin-pya-gu was put together in Unreal Engine by Epic Games. The engine was chosen because it is free to use upfront and is easy to use for non-coders when compared to other game engines (Unity, Cryengine, etc.). Some screenshots from the experience can be seen in Figure 7.

\section{CONCLUSIONS AND FUTURE WORKS}

This paper has presented a workflow that uses photogrammetry to fully create a real-time VR environment. This workflow is ideal for the dissemination and valorisation of heritage spaces. Future work involves optimizing VR performance through lighting and level of detail (LOD) implementations; exploring the relationship between the texture resolution (texel size) and ground sample distance (GSD); and a workflow for capturing the real roughness and specular values of a surface.

\section{ACKNOWLEDGEMENTS}

The authors wish to thank Myanmar's Department of Archaeology, National Museum and Library (DoA) for the opportunity to collaborate in the documentation of this temple. Special thanks also goes to the students from Carleton University and Mandalay Technological University who assisted in the 3D capture during the workshop. This work was supported by the NSERC Create Heritage Engineering grant at Carleton University. 

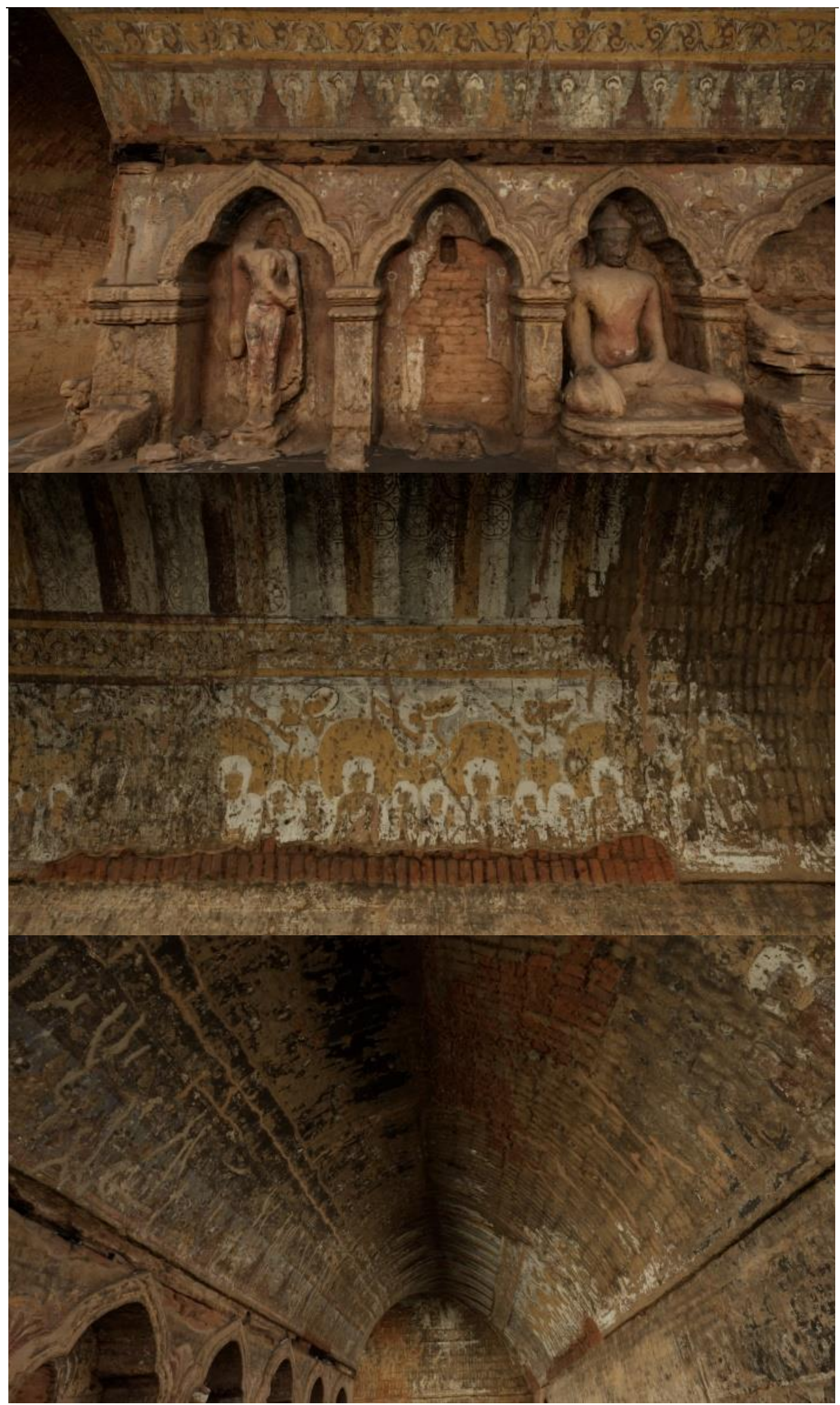

Figure 7. Screenshots from the Myin-pya-gu VR experience 


\section{REFERENCES}

Cignoni, P., Callieri, M., Corsini, M., Dellepiane, M., Ganovelli, F., Ranzuglia, G., 2008. Meshlab: an Open-Source Mesh Processing Tool. Sixth Eurographics Italian Chapter Conference, pp. 129-136.

Croteam, 2014. The Talos Principle, http://www.croteam.com/talosprinciple/ (Last accessed October 2018).

CyArk, 2018. MasterWorks VR: Journey Through History, http://masterworksvr.com/ (Last accessed October 2018).

Curiosity Stream, Experius Vr, Reality Virtual, 2018. Nefertari: Journey to Eternity, https://www.oculus.com/experiences/rift/1491802884282318/ (Last accessed October 2018).

Debailleux, L., Hismans, G., Duroisin, N., 2018. Exploring Cultural Heritage Using Virtual Reality. Lecture Notes in Computer Science, Vol. 10605, pp. 289-303.

Fernández-Palacios, B., Morabito, M., Remondino, F., 2017. Access to Complex Reality-Based 3D Models Using Virtual Reality Solutions. Journal of Cultural Heritage, Vol. 23, pp. 4048 .

Google Developers, 2018. Degrees of Freedom, https://developers.google.com/vr/discover/degrees-of-freedom (Last accessed October 2018).

Graham, K., Fai, S., Dhanda, A., Smith, L., Tousant, K., Wang, E., Weigert, A., 2018. The VR Kiosk. Lecture Notes in Computer Science, Vol. 10605, pp. 324-326.

Hellman, T., Lahti, M., 2018. WindCoE: Photogrammetric 3D Modelling for Virtual Reality. https://www.researchgate.net/project/WindCoE-3 (Last accessed October 2018).

Kersten, T. P., Büyüksalih, G., Tschirschwitz, F., Kan, T., Deggim, S., Kaya, Y., Baskaraca, A. P., 2017. The Selimiye Mosque of Edirne, Turkey - An Immersive and Interactive Virtual Reality Experience using HTC Vive. Int. Archives of Photogrammetry, Remote Sensing \& Spatial Information Sciences, Vol. XLII-5/W1, pp. 403-409.

Kuzmin, V., 2018. Full Photogrammetry Guide for 3D Artists, 80 Level, https://80.lv/articles/full-photogrammetry-guide-for-3dartists/ (Last accessed October 2018).

Lachambre, S., Lagarde, S., Jover, C., 2017. Unity Photogrammetry Workflow, Unity Technologies, https://unity.com/solutions/photogrammetry (Last accessed October 2018).

McDermott, W., 2018. The PBR Guide Volume 2: Practical Guidelines for Creating PBR Textures, Allegorithmic https://www.allegorithmic.com/pbr-guide (Last accessed October 2018).

Oculus, 2018. Oculus Quest, https://www.oculus.com/quest/ (Last accessed October 2018).

Pichard, P., 1995. Inventory of Monuments at Pagan. UNESCO.
Realities.io, 2016. Realities, http://realities.io/ (Last accessed October 2018).

Remondino, F., Nocerino, E., Toschi, I., Menna, F., 2017. A Critical Review of Automated Photogrammetric Processing of Large Datasets. Int. Archives of Photogrammetry, Remote Sensing \& Spatial Information Sciences, Vol. XLII-2/W5, pp. 591-599.

Schewe, $\quad$ J., $2018 . \quad$ Colorspace, https://commons.wikimedia.org/wiki/File:Colorspace.png (Last accessed October 2018).

Slater, M., Wilbur, S., 1997. A Framework for Immersive Virtual Environments (FIVE): Speculations on the Role of Presence in Virtual Environments. Presence: Teleoperators and Virtual Environments, Vol. 6, pp. 603-616.

Statham, N., 2018. Use of Photogrammetry in Video Games: A Historical Overview. Games and Culture, doi.org/10.1177/1555412018786415.

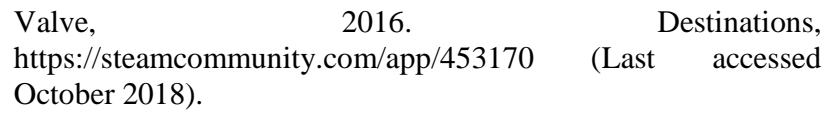

